\title{
5
}

\section{DIVERSIDAD FUNCIONAL $Y$ «DISEÑO PARA TODAS LAS PERSONAS» EN LOS ESTUDIOS DE INGENIERÍA}

\section{PERSONS WITH DISABILITIES AND DESIGN FOR ALL (DFA) IN ENGINEERING STUDIES}

\author{
Yaiza Armas-Cruz \\ yarmas@ull.edu.es
}

Universidad de La Laguna, España

https://doi.org/10.25145/b.innovaull.2019.005 


\section{RESUMEN}

El Proyecto de Innovación Educativa de la Universidad de La Laguna (ULL) a que se refiere este artículo y que lleva por título «El Diseño para Todas las Personas en la docencia en ingeniería. Fase I: visibilizando la diversidad funcional de las personas y su vinculación con los estudios de ingeniería» constituye un primer paso en la incorporación del «Diseño para todos» en el currículo universitario de Ingeniería. En primera instancia se pretende visibilizar la realidad de la diversidad en el ámbito de los estudios de ingeniería, fomentando así la sensibilización y el interés hacia esta realidad que puede convertirse en el desencadenante en los estudiantes de procesos innovadores de conceptualización del propio aprendizaje y la posterior práctica profesional.

Este preliminar acercamiento de los estudiantes de Ingeniería de la ULL a la estrecha vinculación que existe entre su disciplina y la atención a las personas con discapacidad permite aportar la perspectiva socio-económica de la atención a la diversidad, como medio para la integración social y laboral de las personas con diversidad funcional. Asimismo, se pretende que los futuros ingenieros exploren la potencialidad de esta temática como futura especialización profesional circunscrita a un nicho de mercado creciente.

PALABRAS CLAVE: innovación docente; Diseño para Todas las Personas; personas con diversidad funcional; estudios de Ingeniería.

\section{ABSTRACT}

The Educational Innovation Project of the ULL entitled «Design for All People in engineering teaching. Phase I: visualizing the functional diversity of people and their link with engineering studies is a first step in the incorporation of «Design for all (DfA) in the university engineering curriculum. The main prupose is to make visible the diversity in the field of engineering studies, thus promoting awareness and interest in this reality that can become the trigger in the students of innovative processes of conceptualization of their own learning and subsequent professional practice.

This preliminary approach to the close link that exists between engineering studies and care for people with disabilities allows students to contribute to the socio-economic perspective of attention to diversity, as a means to social and labor integration of people. with functional diversity. Likewise, it is intended that future engineers explore the potential of this topic as a future professional specialization circumscribed to a growing market niche. KEYWORDS: teaching innovation; Design for All; people with functional diversity; engineering studies.

\section{INTRODUCCIÓN}

Para conseguir una sociedad "para Todos» que penetre tanto a nivel legal como en la práctica profesional es necesario que se involucren todos los agentes implicados, incluida la educación, y también los que intervienen en la toma de decisiones.

Los futuros profesionales construirán y conducirán la sociedad. Dirigirlos a través de lo que es la diversidad humana y cuanta influencia ejerce sobre el resultado de nuestros planes y acciones provocará un cambio de paradigma que dará forma a un modelo de desarrollo más inclusivo y 
efectivo, porque se originará desde la comprensión de las necesidades y aspiraciones de las personas reales.

La diversidad es una realidad humana como tal y trabajar holísticamente respecto a ella llevará a que los futuros profesionales desarrollen proyectos innovadores más eficientes, eficaces, estéticos e interesantes. Planificar y diseñar para la diversidad humana en un contexto de experiencias abre un gran abanico de posibilidades, desafíos y riqueza. (Suárez Quirós, 2010). En este sentido, EIDD-Design for All Europe (2004) en su Declaración de Estocolmo (C) define el Diseño para Todos como «diseño para la diversidad humana, la inclusión social y la igualdad».

La reflexión acerca de la diversidad funcional de las personas y el «Diseño para Todos» en los estudios de Ingeniería de la Universidad de La Laguna se incorpora por primera vez mediante el Proyecto de Innovación Educativa (PIE) «El Diseño para Todas las Personas en la docencia en ingeniería. Fase I: visibilizando la diversidad funcional de las personas y su vinculación con los estudios de ingeniería». Dicho proyecto se desarrolla en el ámbito de la asignatura básica de rama "Organización y Gestión Empresarial», impartida en el primer curso de la titulación. Se pretende con ello fomentar, ya desde los albores de la trayectoria universitaria, el conocimiento de una temática que, además de su interés humano y social, mantiene una estrechísima vinculación con la práctica profesional de la ingeniería, particularmente en las ramas vinculadas a las tecnologías de la información y la comunicación (Bohman, 2012), así como en las ingenierías Mecánica y Electrónica.

En este artículo se describe el planteamiento y diseño del proyecto, su posterior desarrollo en el aula y los resultados obtenidos. Finalmente, se presentan las conclusiones extraídas del aprendizaje obtenido a partir de la puesta en práctica de esta experiencia de innovación educativa, tanto desde la perspectiva docente como estudiantil.

\section{DIVERSIDAD FUNCIONAL E INGENIERÍA: PLANTEAMIENTO Y DISEÑO DE UNA EXPERIENCIA EDUCATIVA INNOVADORA}

La percepeción de la discapacidad' está cambiando, desde el enfoque tradicional de su conceptualización médica e individual, hasta la reciente perspectiva «biopsicosocial» puesta de manifiesto en la International Classification of Functioning, Disability and Health de la Organización Mundial de la Salud (Barnes, 2011).

El Diseño para Todos es un enfoque, una evolución paradigmática de la manera de pensar, observar y actuar. Dicho enfoque justifica el papel prioritario de la educación universitaria y la posterior práctica profesional, en este caso referido al ámbito técnico (ingeniería).

'Término que en la actualidad está siendo sustituido por «Diversidad Funcional». 
En el contexto del Espacio Europeo de Educación Superior, las universidades adheridas a los acuerdos de Bolonia adquirieron un compromiso con el Diseño para Todos en los currículos (Suárez Quirós, 2010). Si bien Moriña et al. (2017) constatan el importante papel que están desempeñando y el progresivo desarrollo de las oficinas de soporte para la atención a la discapacidad en las universidades españolas, el progreso hacia el objetivo de la plena integración en los currículos es aún incipiente. Según el segundo estudio "Universidad y Discapacidad» elaborado por la Fundación Universia y el Comité Español de Representantes de Personas con Discapacidad (2014), en 2014 el 51\% de las universidades habían incorporado la discapacidad en el diseño de los planes de estudio. Un 29 en la totalidad de los planes de estudio, mientras un $22 \%$ en algunos grados universitarios. El 22\% de las universidades todavía no habían avanzado en la integración de la discapacidad en los planes de estudios universitarios y el $27 \%$ no aportaban información. Dado lo incipiente de este proceso, los estudios de tecnología de la ULL son un «lienzo en blanco» para poner en práctica, al menos de forma exploratoria, esta iniciativa.

El PIE que se presenta constituye una primera y pionera aproximación a la incorporación del "Diseño para todos» en el currículo universitario de Ingeniería en la ULL. Su propósito fundamenteal del PIE que se presenta es atender a la necesidad detectada de que los estudios de ingeniería pongan el foco en sus interrelaciones con y aplicabilidad en la atención a la diversidad funcional de las personas, mediante el Diseño para todos.

Como primer paso es necesario acercar esta realidad a los estudiantes de ingeniería desde una perspectiva socio-económica y científica. Para ello, se propone el análisis de las siguientes temáticas concretas (objetivos específicos):

- Tecnología asistiva. Ingeniería aplicada a la discapacidad.

- Ergonomía y Discapacidad. Aplicaciones desde la ingeniería.

- Estudio del caso: Ingeniería aplicada a la inclusión social educativa.

- Enfermedades raras y viabilidad económica de la aplicación tecnológica.

- Ejemplos concretos y nuevos desarrollos.

Para este propósito se require un enfoque metodológico participativo, colaborativo y centrado en el análisis de experiencias prácticas. Así, el análisis y reflexión sobre la aplicación y el potencial económico y laboral de las interrelaciones entre los estudios y la profesión de ingeniería y el diseño destinado a la integración de personas con discapacidad se plasma en un blog, diseñado y elaborado por los propios estudiantes, que servirá de plataforma de intercambio de información y reflexión sobre el aprendizaje experimentado.

Elaborado de forma colaborativa por el alumnado de la asignatura, dicho blog aborda las temáticas específicas trabajadas previamente en equipos de estudiantes sobre un material aportado por la profesora. Dichas entradas son comentadas por los propios compañeros de asignatura, 
así como por otros estudiantes de otras ramas próximas de ingeniería, a quienes se les cursa invitación a participar voluntariamente mediante los profesores que imparten la asignatura «Organización y Gestión Empresarial» en dichas titulaciones afines (Ingeniería Informática, Ingeniería Civil, Ingeniería de la Edificación, Ingeniería Naval, etc.). Las conclusiones extraídas y plasmadas en el blog son, a su vez, explicadas por los estudiantes a sus compañeros en sesiones presenciales. El blog permitirá, por tanto, el intercambio de impresiones entre todos los estudiantes, favoreciendo así el aprendizaje colaborativo.

Las actividades específicas que se plantean para el desarrollo del PIE son las siguientes:

- Diseño y elaboración del blog, concebido como el soporte para plasmar el aprendizaje colaborativo.

- Análisis y reflexión de los recursos disponibles y la información adicional que será necesario aportar (trabajo en equipo).

- Elaboración de las entradas conforme a los ámbitos temáticos específicos propuestos y los ítems de reflexión planteados por la docente (trabajo en equipo).

- Publicación de las entradas y subsiguiente proceso de reflexión y debate conjunto haciendo uso de la herramienta del blog.

- Co-evaluación del PIE. Reflexión sobre el proceso de aprendizaje experimentado y detección de oportunidades de mejora y avance.

\section{VISIBILIZANDO LA DIVERSIDAD FUNCIONAL DE LAS PERSONAS Y SU VINCULACIÓN CON LOS ESTUDIOS DE INGENIERÍA: DESARROLLO DEL PROYECTO DE INNOVACIÓN EDUCATIVA}

EI PIE se estructura en 4 etapas claramente diferencidas que se desarrollan a lo largo del segundo cuatrimestre del curso 2017/2018 .

Etapa 1. En primer lugar se desarrollará el análisis y reflexión de los recursos e información de partida aportada por la profesora para cada una de las temáticas específicas propuestas. Esto se realizará mediante el trabajo en equipo de los estudiantes con la supervisión y asesoramiento de la profesora.

Etapa 2. A continuación, será necesario buscar y analizar información complementaria que permita a los estudiantes dar respuesta a los «tips» o ítems de reflexión propuestos por la profesora en cada uno de los temas. De nuevo, se trata de un trabajo realizado en equipo.

Una vez cumplimentados estos dos pasos, los equipos temáticos estarán en disposición de diseñar y elaborar una «entrada» al blog en la que aborden, desde su perspectiva de estudiantes de ingeniería, los tópicos de interés que les han sido propuestos en el contexto de la temática de «Diseño para todos e Ingeniería». 
Las etapas 1 y 2 del proyecto se extenderán durante la primera mitad del cuatrimestre.

Etapa 3. A continuación, se iniciará el proceso de publicación semanalmente en el blog de las entradas resultantes. En ese momento, los estudiantesautores tendrán la oportunidad de explicar verbalmente en clase a sus compañeros el contenido, enfoque y aprendizaje incorporado en su entrada, para que, posteriormente, estos puedan iniciar el proceso de respuesta o comentario a las mismas. La etapa 3 tendrá lugar durante la segunda mitad del cuatrimestre.

Etapa 4. Finalmente se llevará a cabo la evaluación conjunta de los resultados del PIE, en términos de aprendizaje colaborativo, satisfacción global, ámbitos de mejora y propuestas de desarrollos futuros.

La tabla 1 recoge el cronograma de las entradas al blog, con el detalle de los recursos de partida y los ámbitos de análisis propuestos a los estudiantes.

\begin{tabular}{|c|c|c|c|}
\hline \multicolumn{4}{|c|}{ TABLA 1: Programación, ReCuRSOS Y ÁmBitos de Reflexión } \\
\hline $\begin{array}{l}\text { Fecha } \\
\text { entrada } \\
\text { al blog }\end{array}$ & Temática & Recursos de partida & Ámbitos de reflexión y análisis \\
\hline 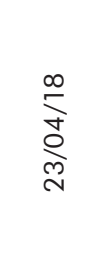 & $\begin{array}{c}\text { Tecnología } \\
\text { asistiva }\end{array}$ & $\begin{array}{l}\text { Fundación Para Los Estudios } \\
\text { De Ingeniería Aplicada A La } \\
\text { Integración Del Discapacitado: } \\
\text { http://www.astus.org/Astus/ } \\
\text { WebAstus10b.nsf/7e9bb520d } \\
\text { ac39350c125772f005c0bbd/ } \\
9 e e 16 c 2 c 149 \text { e95ffc12577e300 } \\
\text { 489f29?OPenDocument. }\end{array}$ & $\begin{array}{l}\text { - Interrelación entre ingeniería y } \\
\text { discapacidad. } \\
\text { - Potencial socio-económico de } \\
\text { este ámbito de aplicación de la } \\
\text { ingeniería como nicho de empleo } \\
\text { y de desarrollo industrial. } \\
\text { - Ramas de ingeniería implicadas. }\end{array}$ \\
\hline 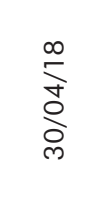 & $\begin{array}{l}\text { Ergonomía y } \\
\text { Discapacidad. } \\
\text { Aplicaciones } \\
\text { desde la } \\
\text { ingeniería }\end{array}$ & $\begin{array}{l}\text { - Instituto de Biomecánica de } \\
\text { Valencia: http://www.ibv.org/. } \\
\text { - Centro Estatal de Autonomía } \\
\text { Personal y Ayudas Técnicas } \\
\text { (CEAPAT):http://www.ceapat. } \\
\text { es/ceapat_01/index.htm. }\end{array}$ & $\begin{array}{l}\text { - Ergonomía y desarrollo de } \\
\text { productos para personas con } \\
\text { discapacidad. } \\
\text { - Otras aplicaciones industriales } \\
\text { de la ergonomía: Prevención, } \\
\text { Salud e Higiene en el Trabajo, etc. }\end{array}$ \\
\hline 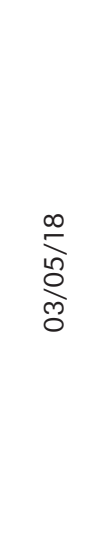 & $\begin{array}{l}\text { Estudio } \\
\text { del caso: } \\
\text { Ingeniería } \\
\text { aplicada a } \\
\text { la inclusión } \\
\text { social } \\
\text { educativa }\end{array}$ & $\begin{array}{l}\text { - Ingeniería aplicada a inclu- } \\
\text { sión social educativa.pdf. } \\
\text {-VIU. Principios de la inte- } \\
\text { gración educativa en el aula } \\
\text { regular: http://www.viu.es/ } \\
\text { principios-de-la-integracion- } \\
\text { educativa-en-el-aula-regular/. } \\
\text { - Villa \& Arnau (2009). Ar- } \\
\text { tículo dedicado a la disca- } \\
\text { pacidad en la Universidad } \\
\text { que recoge las ideas de la } \\
\text { Ponencia presentada en el } \\
\text { Seminario de Telecomunica- } \\
\text { ciones y Discapacidad, orga- } \\
\text { nizado por el Colegio Oficial } \\
\text { de Ingenieros Técnicos de } \\
\text { Telecomunicaciones (COITT). }\end{array}$ & $\begin{array}{l}\text { - Analizar en profundidad una de } \\
\text { las aplicaciones específicas de } \\
\text { la ingeniería en el ámbito dela } \\
\text { discapacidad, como es la plena } \\
\text { integración educativa de las per- } \\
\text { sonas con discapacidad. } \\
\text { - El ámbito universitario y los } \\
\text { equipos interdisciplinares.: nuevas } \\
\text { oportunidades para las personas } \\
\text { con discapacidad y los/as profe- } \\
\text { sionales (p. } 52 \text { del artículo de Villa } \\
\text { y Arnau, 2004). }\end{array}$ \\
\hline
\end{tabular}




\begin{tabular}{|c|c|c|c|}
\hline 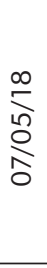 & $\begin{array}{l}\text { «Frágiles». } \\
\text { Enfermeda- } \\
\text { des raras y } \\
\text { viabilidad } \\
\text { económi- } \\
\text { ca de la } \\
\text { aplicación } \\
\text { tecnológica. }\end{array}$ & $\begin{array}{l}\text { Documental «Frágiles, ca- } \\
\text { mino de cristal». Emitido en } \\
\text { Documentos TV, 10/11/2015 } \\
\text { (RTVE.es). }\end{array}$ & $\begin{array}{l}\text { - Cuando la ingeniería no es } \\
\text { viable económicamente. } \\
\text { - Papel de la Responsabilidad } \\
\text { Social Corporativa en el ejercicio } \\
\text { de la ingeniería. }\end{array}$ \\
\hline 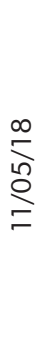 & $\begin{array}{l}\text { "La silla de } \\
\text { Stephen } \\
\text { Hawpking». } \\
\text { Ejemplos } \\
\text { concretos } \\
\text { y nuevos } \\
\text { desarrollos. } \\
\text { Lo que está } \\
\text { por venir. }\end{array}$ & $\begin{array}{l}\text { La silla de Stephen Hawking. } \\
\text { docx (propuesta de análisis } \\
\text { elaborada por la docente a } \\
\text { partir de diversas referencias } \\
\text { en prensa y webs institucio- } \\
\text { nales en relación con este } \\
\text { caso paradigmático de apli- } \\
\text { cación del conocimiento y la } \\
\text { ingeniería a la discapacidad } \\
\text { revolucionaria y trascendental } \\
\text { para la humanidad). }\end{array}$ & $\begin{array}{l}\text { - Reflexionar sobre aplicaciones sor- } \\
\text { prendentes y nuevos desarrollos de } \\
\text { la ingeniería mecánica y electrónica } \\
\text { en el tratamiento y rehabilitación de } \\
\text { diferentes tipos de discapacidad. } \\
\text { - Busquemos otros ejemplos... } \\
\text { - También, por qué no, aplicaciones } \\
\text { para la industria (prevención de } \\
\text { riesgos, salud laboral, etc.). }\end{array}$ \\
\hline 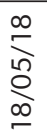 & \multicolumn{3}{|c|}{$\begin{array}{l}\text { Evaluación conjunta de la experiencia de aprendizaje y del desarrollo del PIE } \\
\text { (encuesta y debate grupal). }\end{array}$} \\
\hline
\end{tabular}

Cada una de las temáticas de análisis, y su correspondiente entrada en el blog, fue asignada a un equipo de trabajo. El número total de estudiantes que participaron ${ }^{2}$ fue de 34 , un $46 \%$ de los matriculados en la asignatura en el Grado de Ingeniería Mecánica. Los recursos de partida para el análisis de las temáticas específicas fueron seleccionados por la profesora y facilitados a los correspondientes equipos de trabajo al inicio del cuatrimestre, sin menoscabo de que los estudiantes pudieran completar esta información y documentación de referencia con cuanta otra consideraran oportuna.

\section{RESULTADOS}

En primer lugar, en cuanto a la incorporación de mejoras en la docencia, durante el transcurso de la asignatura se ha aportado a los estudiantes un ámbito de trabajo colaborativo y aprendizaje autónomo y significativo en el que conciliar la transversalidad de las temáticas «Discapacidad» (o «Diversidad Funcional») y «Atención a la diversidad» con su potencial como ámbito de investigación y aplicación práctica en el campo de la Ingeniería.

Estos resultados de aprendizaje se obtienen en el ámbito de la asignatura "Organización y Gestión Empresarial» (básica de rama), permitiendo así a los estudiantes hacer énfasis en el enfoque socio-económico necesario a

${ }^{2}$ La participación de los estudianets fue de carácter voluntario, puesto que esta experiencia innovadora no podía ser computable en la evaluación final de la asignatura. 
tener en cuenta para evaluar la viabilidad de estas interrelaciones como futuro nicho profesional y laboral en el contexto empresarial actual.

La progresión del proceso de aprendizaje queda de manifiesto de forma simultánea a la publicación de las correspondientes entradas en el blog (http://diversidadfuncionalull.blogspot.com/). Dichas aportaciones, así como la reflexión y análisis suscitados a partir del subsiguiente debate, quedaron plasmadas íntegramente y fueron accesibles a través del Aula Virtual de la asignatura, donde estaba referenciado el blog, como herramienta de trabajo colaborativo del alumnado de la asignatura.

Sin embargo, en contra de lo esperado, y como aspecto a mejorar en posteriores desarrollos de esta propuesta de innovación, la participaón de los estudiantes mediante comentarios a las entradas incorporadas por sus compañeros no fue la esperada. Será necesario analizar las causas de la escasa motivación para la réplica para poder corregirla.

Por otra parte, con la finalidad de medir el grado de satisfacción global de los estudiantes con la innovación educativa incorporada a la asignatura, se les pidió su opinión mediante un cuestionario estructurado en el que, además, podían incorporar comentarios abiertos (quejas, sugerencias, opiniones, etc.). La tabla 2 recoge el promedio de puntuación obtenida en cada uno de los ítems considerados:

\begin{tabular}{|l|c|}
\hline \multicolumn{2}{|c|}{ TABLA 2.: ENCUESTA DE VALORACIÓN } \\
\hline \multicolumn{1}{|c|}{ Ítems (escala: 0-10) } & Media \\
\hline La idea del proyecto es apropiada. & 8,1 \\
\hline La puesta en práctica del proyecto por medio del diseño de un blog es adecuada. & 6,7 \\
\hline $\begin{array}{l}\text { El modo de participación de los estudiantes mediante entradas al blog y comentarios } \\
\text { al mismo es oportuno. }\end{array}$ & 7,1 \\
\hline Preferiría otro modo de analizar la «diversidad funcional». & 7,5 \\
\hline Las temáticas elegidas para las entradas del blog son adecuadas. & 8,4 \\
\hline $\begin{array}{l}\text { Preferiría tratar otras temáticas referentes a «diversidad funcional» diferentes a las } \\
\text { que se han tratado. }\end{array}$ & 4,7 \\
\hline La profesora ha desempeñado su papel promotor y dinamizador de forma adecuada. & 8,8 \\
\hline En qué medida contribuye este proyecto a mi aprendizaje. & 5,6 \\
\hline Utilidad de los contenidos aprendidos en mi futura práctica profesional. & 6,5 \\
\hline Participar en este PIE me ha gustado. & 5,4 \\
\hline $\begin{array}{l}\text { Participar en este PIE me resulta interesante pero me supone un gasto de tiempo que } \\
\text { no me compensa. }\end{array}$ & 6,6 \\
\hline Considero que este proyecto es conveniente para la docencia en ingenierías. & 7,6 \\
\hline \multicolumn{1}{|c|}{ Tasa respuesta: $23 \%$} \\
\hline
\end{tabular}

Destacan como muy positivamente valorados la idea del proyecto, así como la selección de temáticas específicas a analizar. La puntuación más baja, si bien aún satisfactoria, corresponde a la percepción de los estudiantes sobre la contribución que este PIE realiza a su propio aprendizaje, así como el disfrute durante su desarrollo. 


\section{CONCLUSIONES}

Del análisis de los resultados del proyecto anteriormente expuestos con la información extraída tanto de las aportaciones de los estudiantes a través del blog, como de sus respuestas en el cuestionario de evaluación con preguntas cerradas y abiertas, así como en los debates abiertos y conjuntos en el aula, se desprenden las conclusiones que se presentan a continuación.

Se detecta la necesidad de analizar las causas de la desmotivación de los estudiantes a la hora de comentar las entradas de sus compañeros en el blog. Sin embargo, en el debate abierto y conjunto en la clase presencial el nivel de participación es muy significativo. Una vez sean analizadas dichas causas, cabría también cuestionar la idoneidad o no de modificar el planteamiento didáctico para vincular la participación de los estudiantes en el PIE a la calificación de la asignatura, y no únicamente al proceso colectivo de aprendizaje transversal.

Se debe, asimismo, profundizar en el conocimiento de la percepción que los estudiantes tienen del PIE en términos cualitativos, dado que estos otorgan una puntuación muy moderada a la contribución de este PIE a su proceso individual de aprendizaje. Dicho análisis de sus percepciones debe guiar el planteamiento futuro de continuidad y/o avance de esta propuesta de innovación educativa. En este sentido, se constata la necesidad de materializar mejor las importantes aportaciones que el aprendizaje colaborativo de aspectos de transversalidad como es la diversidad funcional puede reportar a los estudiantes de ingeniería, no solo durante su proceso de formación sino en su posterior ejercicio profesional. A priori se pueden plantear medidas como, por ejemplo, posibilitar la participación activa de los estudiantes en la selección de las temáticas específicas a analizar y/o en la selección de los recursos de partida para la reflexión.

Por otra parte, en el ámbito de la convocatoria de proyectos de innovación educativa de la ULL se nos invita a los docentes a reflexionar sobre el potencial de la propuesta de innovación para convertirse en una práctica docente estable, así como la extrapolación de los resultados a otras materias y titulaciones. En este sentido, la progresiva incorporación de contenidos de atención a la diversidad funcional es un requisito del EEES, por lo que esta experiencia "piloto» puede servir como base para testar su incorporación y podrá ser replicable y mejorable en otros ámbitos de ingeniería, así como en otras titulaciones. La metodología podría ser semejante, si bien sería necesario adaptar el contenido específico de los temas a la disciplina correspondiente.

Finalmente, la reflexión sobre los posibles avances futuros para la consolidación de esta propuesta de innovación educativa se centrará en los siguientes aspectos:

a) Abordar la Fase II «Conexión con la realidad»:

- Conocimiento de instituciones orientadas a la vinculación entre ingeniería y discapacidad. 
- Delimitación de servicios, productos, proyectos de investigación, etc. concretamente destinados al Diseño para todas las Personas con la directa participación de las diferentes ramas de ingeniería que nos competen.

- Incorporar el testimonio personal de técnicos de la materia que aporten la perspectiva aplicada (ej.: Colegios técnicos oficiales, Once, Apanate, Aspronte, etc.).

b) Difusión de la iniciativa y el conocimiento obtenido con ella por los estudiantes y el profesorado, trascendiendo del ámbito interno (ULL) con el apoyo de herramientas de alcance más generalizado que nuestro campus virtual, como son las redes sociales.

c) Incorporación de un cuadro de mandos con indicadores de control colaborativo de la evolución del proyecto (variables de percepción, índices de satisfacción, etc.).

\section{REFERENCIAS BIBLIOGRÁFICAS}

BARNES, C. (2011). Understanding disability and the importance of design for all. Journal of Accessibility and Design for All (JACCES) 1(1): 55-80. DOI: http://dx.doi. org/10.17411/jacces.v1i1.81.

Bohman, P.R. (2012). Teaching Accessibility and Design-For-All in the Information and Communication Technology Curriculum: Three Case Studies of Universities in the United States, England, and Austria. All Graduate Theses and Dissertations. 1369. Available at.: https://digitalcommons.usu.edu/etd/1369.

EIDD-Design for All Europe (2004). The EIDD Stockholm Declaration( $)$. Adopted on 9 May 2004, at the Annual General Meeting of the European Institute for Design and Disability in Stockholm. «Good design enables, bad design disables». Disponible en: http:// dfaeurope.eu/what-is-dfa/dfa-documents/the-eidd-stockholm-declaration-2004/.

Fundación Universia y Comité Español de Representantes de Personas con Discapacidad (2014). II Estudio «Universidad y Discapacidad». Disponible en: https://www. fundacionuniversia.net/ii-estudio-universidad-y-discapacidad/.

Moriña, A.; López-Gavira, R.; Morgado, B. (2017). How do Spanish disability support offices contribute to inclusive education in the university? Disability \& Society, 32 (10): 1608-1626. https://doi.org/10.1080/09687599.2017.1361812.

Suárez Quirós, J. (coord.) (2010). Formación Curricular de Diseño para Todos en Ingeniería Industrial. Ed.: Instituto de Mayores y Servicios Sociales (IMSERSO) y Fundación ONCE. ISBN: 978-84-8893-44-6.

Villa Fernández, N.; Arnau Ripollés, M.S. (2009): Las Personas con discapacidad en la Universidad. Antena de Telecomunicación, septiembre 2009: 48-54. Disponible en: http://www.javeriana.edu.co/documents/245769/339704/Discapacidad_en_ la_Universidad.pdf/c48247e0-42d4-46b0-adf0-f0b8f78fa083. 